\title{
Stress Intensity Effect on Solid State Oxidation of Ni-Cr Alloy with Different Chromium Concentrates*
}

\author{
Ismail TIRTOM**, Nishith Kumar DAS** and Tetsuo SHOJI** \\ ** Tohoku University, Mechanical Engineering \\ 6-6-1, Aoba, Aramaki, Aoba-ku, Sendai, Japan \\ E-mail:itirtom@rift.mech.tohoku.ac.jp
}

\begin{abstract}
Ni-base alloy is widely used in light water reactor component and the recent study has shown stress corrosion cracking (SCC). Over the years various attempts have been made to obtain mechanism of SCC but it still require more fundamental study to understand clearly. This study presents an approach based on the multiscale modeling, to assess the influence of alloy composition and stress intensity on the initial stage of solid state oxidation of the $\mathrm{Ni}-\mathrm{Cr}$ alloy. The multiscale modeling considers different length scales such as finite element method (FEM) / quasi-continuum (QC) / quantum chemical molecular dynamics (QCMD), for analyzing crack tip molecular domain. The compact tension (CT) specimen of alloy 600 has been loaded for stress intensity, after that the micro region has chosen for the QC model which is a combination of continuum and atomic method. Finally, the deformed atomic position has picked for the QCMD simulation with some water molecules. The simulated results show that the chromium segregates faster than nickel atoms from the surface and make preferential bonding with oxygen. The preferential bonding forms a passive film. Applied stress intensity deformed the structure which may increase the atomic distance. As distance increases the absorption of water molecule or $\mathrm{OH}$ or oxygen into lattice increases. The stress intensity raises the crack tip solid state oxidation that may enhance SCC initiation.
\end{abstract}

Key words: Solid State Oxidation, Stress Intensity, Ni-Cr Alloy

\section{Introduction}

The life extension of aging nuclear power plant is a challenge due to the many and varied phenomena which influence and affect the properties of the materials. The issue of understanding materials behavior exposed to extreme environment. Stress Corrosion Cracking (SCC), i.e., Environmentally Assisted Cracking (EAC) is one of the major failure causes of power plants and components. The crack initiation and growth of EAC are dependant to the combination of materials, environments and stress/strain state [1,2]. Most of the proposed EAC mechanisms deal with mass transfer behavior through and/or beneath the oxide film at the crack tip region. There have been many studies on oxide film in the environments and the relation between oxidation and EAC [3-6]. However oxidation of alloys is a great concern in engineering, to study about oxidation is necessitate knowledge of basic chemistry and material science with using the statistical science concepts because

*Received 16 Nov., 2009 (No. t4) [DOI: 10.1299/jmmp.4.931]

Copyright () 2010 by JSME 
of the process complexity. Positions of millions of atom are rearranging depend on many parameters. On the other hand, atomic diffusion behavior for each different kinds of Bravais lattice is shown different characteristic with various elements. Therefore, it is very difficult to establish a common model for oxidation in metals and alloys. Recently developing computational materials science methods which are based on quantum mechanics, it is possible now to observe atomic behaviors in very tiny spatial and time scales with the high accuracy.

Ni-base alloys are widely used in light water reactor component and stress corrosion cracking (SCC) has been found in pressurized water reactor (PWR) components such as vessel head penetrations and bottom mounted instrument nozzles fabricated with Alloy 600 and its weld metals [7,8]. Ni-base alloys composed of several elements among them nickel and chromium are the major ingredients. X-ray photoelectron spectroscopy (XPS) study of $\mathrm{Ni}-\mathrm{Cr}$ alloy examined that $\mathrm{Cr}$ is oxidized preferentially to $\mathrm{Cr}_{2} \mathrm{O}_{3}$ initially but that $\mathrm{Ni}$ in the alloy is oxidized more rapidly to $\mathrm{NiO}$ than Ni metal [9]. Alloy 600 XPS study found the chromite-rich inner layer due to inward oxygen penetration and simultaneously with outward migration of nickel [10]. However, some theoretical and experimental studies have been carried out on $\mathrm{Ni} / \mathrm{H}_{2} \mathrm{O}$ and oxygen preadsorbed $\mathrm{Ni} / \mathrm{H}_{2} \mathrm{O}$ to understand water dissociation and adsorption phenomena $[11,12]$. Over the years various attempts have been made to obtain mechanism of SCC but it still require more fundamental study to interpret clearly. This study presents an approach based on the multiscale modeling, to assess the influence of alloy composition and stress intensity $(\mathrm{K})$ on the initial stage solid state oxidation of the Ni-Cr binary alloy. The multiscale modeling considers different length scales such as FEM, QC-method, and QCMD, for analyzing crack tip molecular domain. In this study, it is investigated that the initial stage of the water Ni-Cr alloy interaction at the atomic resolution by tight binding methods under the far most loading effect using multiscale approach which is one of the critical issue on stress corrosion cracking models based on slip dissolution/oxidation processes. It will be detailed later that results show loading effect on oxidation and strong interaction of oxygen and chromium.

\section{Computational Methods}

Recent improvements in computational materials science make it possible that to simulate materials behavior at different scales; from electrons to the structural component. However most critical part recognized that atomic scale calculations because it has the capability to see individual atomic movements which is very difficult to see with in-situ experiments. Atomic modeling methods may be classified in two class simply as quantum mechanics based and classic mechanics based. Classic mechanics models are based on Newton laws of motion using interatomic potentials of location. These atomic models can be used with millions of atoms and relatively long time range however not possible to see chemical reactions. On the other hand, quantum mechanics based models can be used to simulate chemical reactions of atoms (electron interactions) however with less number of atoms and short time range. Therefore, advantageous of different methods can be used to solve a particular problem which shows different characteristic behaviors on different scales by multiscale modeling.

Multiscale methods are newly developed tools to analyze materials issues. It is an important part of the multiscale modeling to make linkage in between different scales. SCC is a difficult task to assign multiscale methods from atom to component. We have applied a hierarchical computational model that consists of three different methods to simulate initial stage of oxidation for SCC problem. The details calculation procedure of FEM, QC, and QCMD methods were given elsewhere $[13,14]$. The crack tip is a few nanometers wide and chemical reaction is being performed by tight-binding QCMD. This region plays an 
essential role for primary stage SCC initiation. The slab models for this method is consisted of 48 metallic atoms consider an average size model with single layer six water molecules placed on surface. We have substituted different chromium (10, 15 and 20 percentages) atoms in the Ni surface with or without applying stress intensity. The initial structure for this calculation is shown in Fig. 1. Total number of simulation step was 5000 where each step was 0.2 femtosecond (fs) with the Verlet algorithm. The ensemble for this simulation was constant volume and constant temperature (NVT) and the temperature was controlled by using scaling the atom velocities. Temperature was considered $598 \mathrm{~K}$ for PWR condition and pressure was atmospheric.

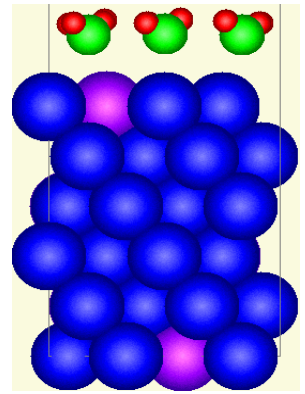

(a)

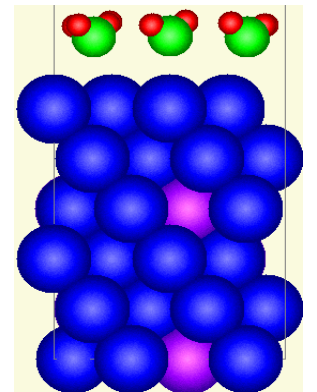

(b)

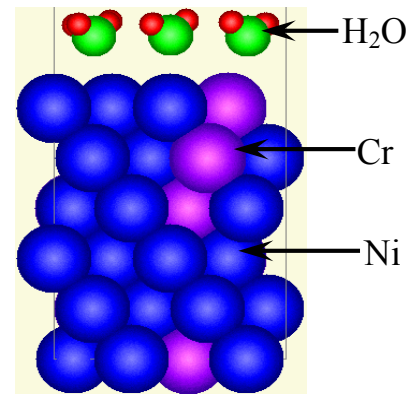

(c)

Figure 1. Ni-Cr (111) initial surfaces with different chromium concentrations; (a) 10\%, (b) $15 \%$ and (c) $20 \%$

\section{Results and Discussion}

The interaction of water with a metal surface is of fundamental interest in studying catalysis, corrosion and passivation phenomena. There are several studies discussed about water absorption on metal surface whether molecularly or dissociatively absorbed [15-17]. Additionally, Pache et al. have demonstrated that water adsorbs molecularly on nickel surface up to $260 \mathrm{~K}$ [18]. In PWR environment, metal water interaction resulted in dissociation of water molecules. Water molecules moved towards the metal surface with progressing of reaction.

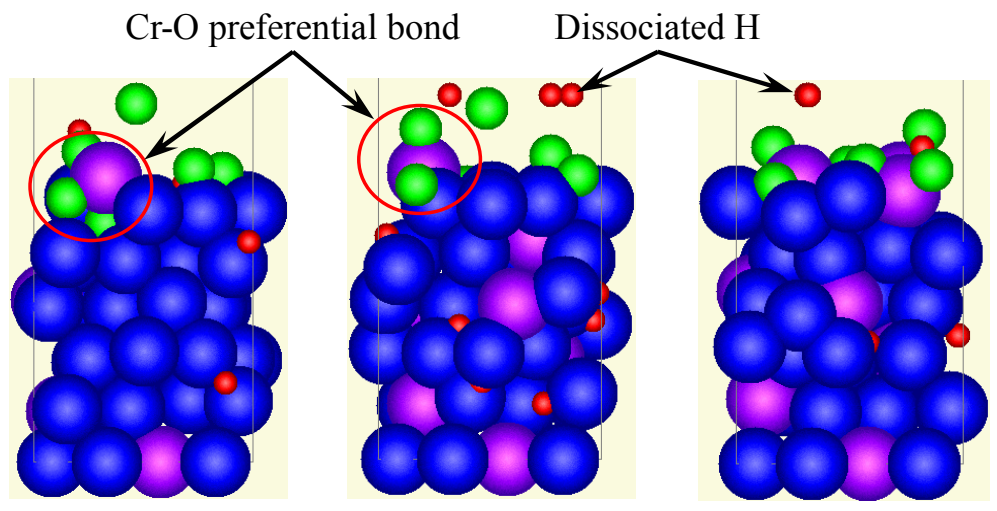

Figure 2. Simulated surface morphologies for without loading case at $5000^{\text {th }}$ step for 10,15 and $20 \% \mathrm{Cr}$ models respectively

After a few hundreds of fs, the interaction assisted to dissociate water molecules into $\mathrm{OH}, \mathrm{H}$ or O. Subsequently, dissociated hydrogen diffused quickly in the surfaces through the interstitial hollow sites due small atomic size. Some of the hydrogen remain above the 
surface that enable to form hydrogen molecule. Hydrogen molecule or dissociated hydrogen above the surface dissolves into the solution. Oxygen atoms penetrate metal surfaces quite slowly as compared to hydrogen.

Fig. 2 shows the various kinds of simulated surface morphologies. The oxygen concentration is evidently high around the chromium and it denotes the commencement of passivation. It is well known that chromium is a passivation promoter and makes preferential bond with the oxygen. From the simulation results it is observed the higher rate of chromium segregation than nickel. Chromium cohesive energy is low in comparison to nickel as well as diatomic bond energy also. These may benefit for the segregation of chromium. As a result, $\mathrm{Ni}$ enriched inner oxide layer make possible to develop at the primary stage. The result is consistent with theoretical and experimental studies $[19,20]$. It is difficult to identify oxygen diffusion precisely from the surface morphologies. Hence, Fig. 3 illustrates for average oxygen diffusion along the z-axis of various kind of surfaces. Stress intensity certainly increases oxygen diffusivity. The higher chromium concentration reduces the oxygen diffusivity which indicates chromium decelerates the oxidation process. Applied $\mathrm{K}$ has distorted surfaces and heightens the interatomic distances that might help to travel oxygen (Fig. 4).

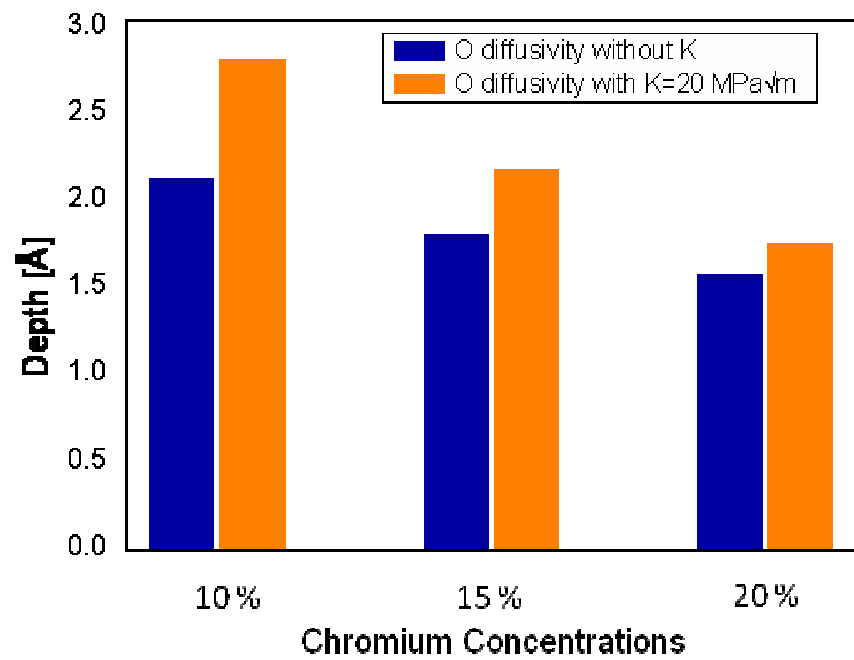

Figure 3. Average oxygen diffusivity comparison in between different chromium concentrations and stress intensities

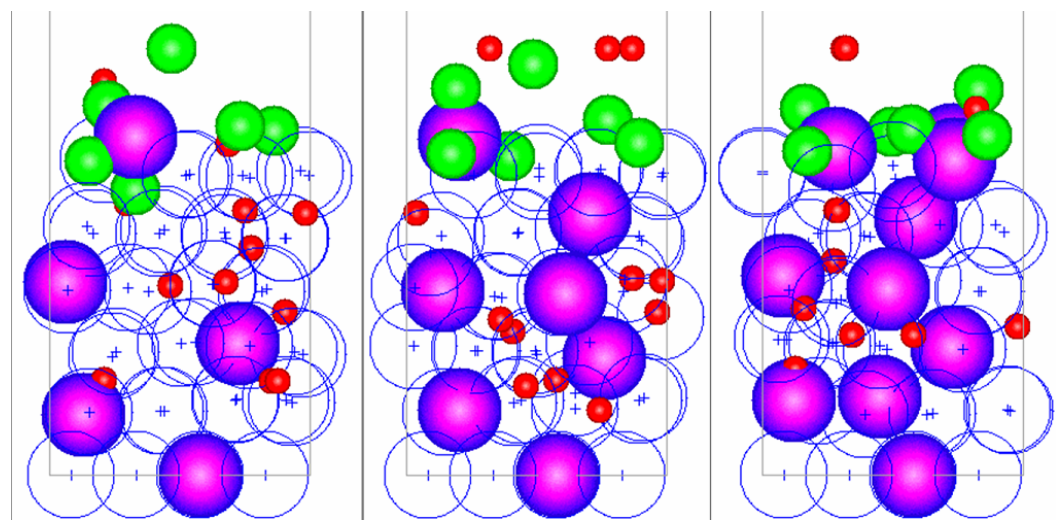

Figure 4. Simulation results under the loading: Atomic configuration of three $(10,15$ and $20 \% \mathrm{Cr}$ concentration, respectively) models at $5000^{\text {th }}$ step 
Average oxygen diffusivity decreases with the increasing of chromium percentage in the surfaces. Oxygen trap around the chromium due to higher binding energy as a result, it decelerates oxygen diffusivity gradually. So, the localized stress intensity empowers the early stage oxidation process and chromium lessening the oxygen mobility.

\subsection{Average Atomic Charge}

It was carried out the average atomic charge progression by Mulliken population [21, 22]. The present study has shown that the water molecules dissociatively adsorbed on the metal surface due to high temperature environments subsequently dissociated species diffuse in the metal surface. Chromium and hydrogen shows the positive in charge and oxygen is negatively charged at the beginning stage of calculation, as shown in Fig. 5 . Water molecules adsorbed by the surfaces and it started to dissociation after a few hundreds of fs. Dissociated water molecules produced $\mathrm{O}, \mathrm{OH}$, and $\mathrm{H}$. Several dissociated hydrogen atoms passed through the structure quickly and some of them reside above the surface. Deeply diffused hydrogen changed into negatively charged by taking electron from the metal atoms because the metal outer orbitals excess electron can be able to donate to another receiver.

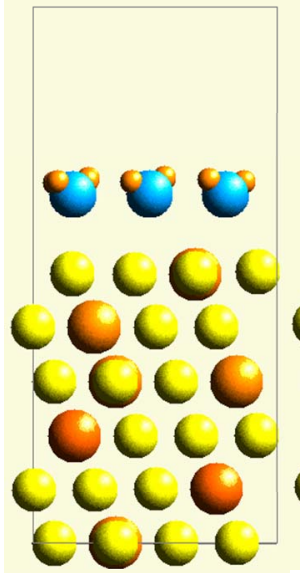

initial

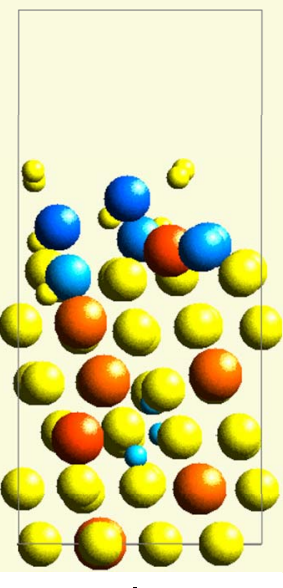

$1350^{\text {th }}$ step

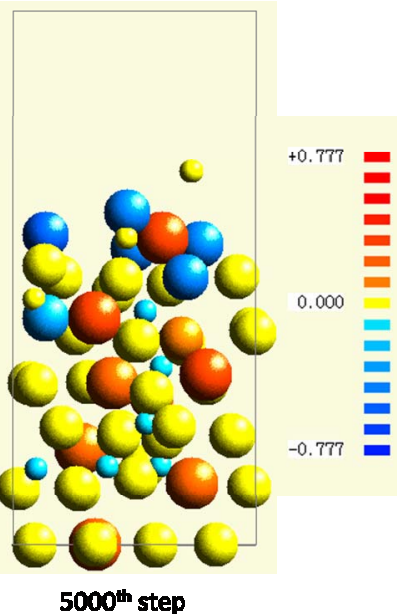

$\mathbf{5 0 0 0}^{\text {th }}$ step

Figure 5. Atomic charge distribution at several steps during the course of $15 \% \mathrm{Cr}$ percentage, under loading model

Several studies demonstrated that it is possible to be negative in charge of hydrogen in metal and it enable to accelerate oxidation [19, 23-25]. The result is good agreement with the indication of earlier studies. The electron sharing process makes the metal atom positively charged and chemically active for reaction consequently slightly columbic repulsion develops in between them. Thereafter, when the dissociated oxygen or $\mathrm{OH}$ comes into contact with them that helps to form bond by exchanging electron. This interaction assists to pull out the metallic atoms from the place and make a vacancy subsequently oxygen atoms penetrates into the surface. The process accelerates the surface oxidation process. Notable negatively charged oxygen atom make favorable bond with the extremely positively charged chromium atoms which decelerates the oxygen mobility when it traps around chromium. This mechanism initiates the passive film formation on the surface.

\section{Conclusion}

The stress intensity effect on initial stage oxidation of Ni-Cr (111) binary alloy surface has been studied by multiscale modeling. QCMD method has applied to analyze reaction 
dynamics at the crack tip molecular domain. External load, which is corresponded to different stress intensities, applied CT-specimen computational model which has very fine mesh around crack tip has been calculated by FEM. Micrometer scale deformations at the vicinity of the crack tip are obtained. These deformation values are used as input for the prepared, a crack included, micrometer size QC model. After the completion of QC calculations, obtained deformed atomic positions in the crystal structure are used to construct for oxidation mechanism analysis. The QCMD simulated results examined that the chromium segregated faster than nickel atoms and make preferential bonding with oxygen. The preferential bonding originates a passive film formation. Applied stress intensity deformed the structure which may increase the atomic distance. This distance heightens the absorption of water molecule or $\mathrm{OH}$ or oxygen into lattice. Substitution of chromium into the surface decelerates oxygen diffusivity due to surface passivation. In conclusion, the stress intensity raises the crack tip solid state oxidation that may enhance at the beginning stage of SCC initiation.

\section{References}

1. Shoji, T., Suzuki, S., and Ballinger, R. G., "Theoretical Prediction of SCC Growth Behavior-Threshold and Plateau Growth Rate", Proceedings of Seventh International Symposium on Environmental Degradation of Materials in Nuclear Power Systems-Water Reactors, Breckenridge, CO, pp. 881-889, 1995.

2. Ford, F. P., "Mechanisms of Environmentally-Assisted Cracking", Int. J. Pressure Vessels Piping, 40(55), pp. 343-362, 1989.

3. Kim, Y.-J., "Characterization of the oxide film formed on type 316 stainless steel in $288{ }^{\circ} \mathrm{C}$ water in cyclic normal and hydrogen water chemistries", Corrosion, 51(11), 849-860, 1995.

4. Wang, S., Takeda, Y., Shoji, T., and Kawaguchi, N., "Observation of the oxide film formed in high temperature water by applying electroless Ni-P coating", Journal of Nuclear Science and Technology, 41(7), 777-779, 2004.

5. Stephen E. Ziemniak, Michael Hanson. Corrosion behavior of NiCrFe Alloy 600 in high temperature, hydrogenated water. Corrosion Science, 2006, 48: 498-521.

6. Terachi, T., Fujii, K., and Arioka K., "Microstructural characterization of SCC crack tip and oxide film for SUS 316 stainless steel in simulated PWR primary water at $320^{\circ} \mathrm{C}$ ", Journal of Nuclear Science and Technology, 42(2), 225-232, 2005.

7. Scott, P. M. and Benhamou, C., In Proceedings of the 10th International Symposium on Environmental Degradation Materials Nuclear Power Systems - Water Reactors, NACE, CDROM, 2001.

8. Scott, P. M. and Combrade, P., In Proceedings of the 11th International Symposium on Environmental Degradation Materials Nuclear Power Systems - Water Reactors, ANS, CDROM, 2003.

9. Jeng, S. P., Holloway, P. H. and Batich, C. D., Surface Science, vol. 227, 278-290, 1990.

10. Ziemniak, S. E. and Hanson, M., Corrosion Science, vol. 48, 498-521, 2006.

11. Sebastiani, D. and Site, L. D., Journal of Chemical Theory and Computation, vol. 1, 78-82, 2005.

12. Wang, G. C., Wao, S. X. and Bu, X. H., Journal of Catalysis, vol. 244, 10-16, 2006. 
13. Tirtom, I., Das, N. K., Suzuki, K., Ogawa, K. and Shoji, T., Multiscale modeling approach to stress corrosion cracking, Proceedings of the 2008 International Congress on Advances in Nuclear Power Plants, 2008, Anaheim, California, USA, pp 2081-2089.

14. Das, N. K., Tirtom, I. and Shoji, T., Materials Chemistry and Physics, vol. 122, $336-342,2010$

15. Gakkagher, M. E., Haq, S., Omer, A. and Hodgson, A., Surface Science, vol. 601, 268-73, 2007.

16. Madey, T. E. and Netzer, F. P., Surface Science, vol. 117, 549-560, 1989.

17. Pozzo, M., Carlini, Rosei, G., R. and Alfe, D., Journal Chemical Physics, vol. 126, 164706, 2007.

18. Pache, T., Steinrück, H. -P., Humber, W. and Menzel, D., Surface Science, vol. 224, 195-214, 1989.

19. Das, N. K., Suzuki, K., Ogawa, K. and Shoji, T., Corrosion Science, vol. 51, 908-913, 2009.

20. Terachi, T., Fuji, K., and Arioka, K., Journal of Nuclear Science and Technology, 42, $225,2005$.

21. R. S. Mulliken, Journal of Chemical Physics, Vol. 23 (1955), 1833.

22. K. Suzuki, Y. Kuroiwa, S. Takami, M. Kubo, A. Miyamoto and A. Imamura, Solid State Ionics, vol. 152-153, 273-277, 2002.

23. Das, N. K., Suzuki, K., Takeda, Y., Ogawa, K. and Shoji, T., Corrosion Science, vol. $50,1701-1706,2008$.

24. Wallinder, D., Hörnlund, E., and Hultquist, G., J. Electrochem. Soc., Vol. 149, B393, 2002.

25. Tveten, B., Hultquist, G., and Wallinder, D., Oxid. Met., Vol. 55, 279, 2001. 